\title{
La necesidad de arte y su papel como instrumento para la construcción de la memoria colectiva
}

\author{
The necessity of art and its role as an instrument for \\ the construction of collective memory
}

\section{LuCILA URdA PEÑA}

Profesora Asociada. Departamento de Urbanística y Ordenación del Territorio. Escuela Técnica Superior de Arquitectura. Universidad Politécnica de Madrid

lucilaurda@hotmail.com

Recibido: 09/11/2015

Aceptado: 08/02/2016

\begin{abstract}
Resumen
La ausencia de relación entre los ciudadanos y su ciudad de residencia es un hecho en la sociedad contemporánea. El modo de vida utilitarista, que procura una economía del tiempo y una búsqueda de beneficios económicos a corto plazo no favorece la generación de espacios compartidos de convivencia en las ciudades. Sin embargo, la aparición de ciertos movimientos artísticos espontáneos en determinados colectivos urbanos a principios del siglo XXI parece anunciar nuevos modos de gobernanza para las ciudades. La necesidad de arte ha sido un tema sobre el que han reflexionado pensadores y filósofos desde principios del siglo XX y su papel como instrumento constructor de la memoria colectiva vuelve a ser motivo de debate y discusión en el siglo XXI.
\end{abstract}

Palabras clave

Gobernanza, identidad, arte urbano, espacio público. 


\begin{abstract}
The absence of relation between the citizens and their cities is a fact in the contemporary society. The utilitarian way of life does not provide the creation of shared spaces for the communities in urban spaces. However, the appearance of artistic spontaneous movements in urban contexts at the beginning of century 21st century seems to announce new ways of gobernance for the cities. Philosophers have been reflecting about the necessity of art from the beginning of 20th century. The role of the art as an instrument for the construction of the collective memory returns to be reason for debate and discussion in 21st century.
\end{abstract}

\title{
Keywords
}

Governance, identity, urban art, public space.

Referencia normalizada: URDA PEÑA, LUCILA (2016): “La necesidad de arte y su papel como instrumento para la construcción de la memoria colectiva". Arte y Ciudad. Revista de Investigación, no 9 (abril), págs. 91-104. Madrid. Grupo de Investigación Arte, Arquitectura y Comunicación en la Ciudad Contemporánea, Universidad Complutense de Madrid.

\section{La necesidad de arte.}

La pérdida del vínculo del ciudadano con el ámbito urbano que le es inmediato, el espacio que utiliza y que, al fin y al cabo, rodea su intimidad, es una realidad creciente en las grandes ciudades.

Ya en los años 60, Jane Jacobs nos alertaba de la muerte de la vida ciudadana en las grandes ciudades. En su libro Muerte y vida de grandes ciudades describe una apatía ciudadana alertando de las consecuencias que podría provocar en la sociedad urbana.

Es posible que nos hayamos convertido en una gente tan abúlica que ya no nos importe cómo funcionan las cosas, sino únicamente la primera, rápida y externa impresión que nos dan. De ser así, poca esperanza hay para nuestras ciudades, o para cualquier otra cosa en nuestra sociedad. Pero no creo que sea así (Jacobs, 1961).

Realmente el grado de interés sobre nuestro entorno urbano depende del nivel de conocimiento de las causas de sus transformaciones y de la posibilidad de participación en los procesos. La carencia de información y la falta de 
capacidad del ciudadano para intervenir en la ciudad desvinculan al usuario de su contexto urbano. Sin embargo, las herramientas que Jane Jacobs propone para lograr una ciudad viva, "que funcione" son de sentido común: el contacto entre vecinos, la diversidad del comercio, la valoración del patrimonio o la concentración de actividades.

Pese a que en el libro se recogen algunas reflexiones sobre el funcionamiento del espacio público, lo que preocupa y trasciende de la obra de Jacobs es la pérdida de identidad del vecindario y, por tanto, las consecuencias que se perciben en el uso del espacio público. La falta de identidad colectiva en los barrios hoy en día es una realidad que afecta directamente al uso y al mantenimiento de los lugares de uso común. La ausencia de un proyecto colectivo, o de ciertos intereses comunes desencadena un desinterés general del vecino por su barrio.

El interés sobre la identidad y la falta de identidad es un asunto sobre el que se lleva escribiendo desde principios del siglo XX. Ya Charles Baudelaire, en su breve escrito El pintor de la vida moderna (Baudelaire, 1863) habla de la modernidad como aquello que pasa, que no permanece, que carece de valores estables. "La modernidad es lo transitorio, lo fugitivo, lo contingente, la mitad del arte, cuya otra mitad es lo eterno y lo inmutable". Y vuelve a insistir: “(...) Ha buscado por todas partes la belleza pasajera, fugaz, de la vida presente, el carácter de lo que el lector nos ha permitido llamar la modernidad".

$\mathrm{Su}$ reflexión permite extraer conclusiones sobre la superficialidad que parece que va invadiendo los modos de hacer contemporáneos. La superficialidad es consecuencia de la falta de experiencias y de vivencias profundas a causa de la compleja vida urbana. A raíz de esa especulación, Walter Benjamin escribió sobre la importancia de la experiencia como el modo de enriquecer su memoria, tanto privada como colectiva.

La experiencia es un hecho de tradición, tanto en la vida privada como en la colectiva. La experiencia no consiste principalmente en acontecimientos fijados con exactitud en el recuerdo, sino más bien en datos acumulados, a menudo de forma inconsciente que afluyen a la memoria (Benjamin, 1939).

Existe un vínculo de la experiencia con los recuerdos $\mathrm{y}$, finalmente, con la memoria. La memoria es también la que construye nuestra historia y, de algún modo, nuestra identidad, por tanto la experiencia colectiva es la que construye una memoria colectiva que se consolida con la vida en comunidad. 
Christine Boyer en su libro The city of collective memory (1996) hace un repaso histórico sobre los primeros autores interesados en la pérdida de memoria colectiva, en él menciona a pensadores de la literatura contemporánea que reflexionan sobre la crisis del imaginario común y sus posibles causas y soluciones. Según Boyer, Walter Benjamin achaca a la vida moderna un problema de amnesia y, por tanto, de pérdida de valores. La recuperación de la memoria colectiva sólo es posible para Walter Benjamin a través de una relación normal entre pasado y presente con técnicas para recuperar memorias olvidadas y trazas escondidas.

En su repaso, Boyer recurre a soluciones aportadas por varios autores. Sigmund Freud propone acudir al mundo de los sueños para restaurar la continuidad en la realidad a partir de sus significados fragmentarios, Henry Bergson considera que a través de la representación (proceso de recolección más percepción) se encuentra el modo de la expresión de estados internos. Para Maurice Halbwachs las raíces de la memoria colectiva están en las experiencias sociales y asociadas con marcos espacio-temporales. Gilles Deleuze afirma que pensar quiere decir estar embebido en el estrato del tiempo actual. Cualquiera de las soluciones planteadas vincula al individuo con su experiencia personal asociada a un lugar o a un grupo de personas en un contexto temporal.

Pese a que parece que la pérdida de memoria colectiva es un problema antiguo que condujo a discusiones y a debates a principios del siglo $\mathrm{XX}$, la realidad es que, en el siglo XXI no solo no ha desaparecido sino que, probablemente, se ha agravado. Las reivindicaciones sectoriales de carácter social de grupos con intereses muy acotados (mujeres, homosexuales, grupos marginales, antisistema, etc.) han alcanzado logros con respecto a cada una de sus pretensiones pero por otra parte han imposibilitado el sentimiento común de grupos heterogéneos de personas con una identidad común a pesar de sus diferencias.

La urgencia de conseguir un tratamiento justo e igualitario para todos deja de lado y, en segundo plano, las reivindicaciones que vinculan al ciudadano con su espacio urbano de referencia y, solo a raíz de situaciones extraordinarias $^{1}$, se producen vínculos de carácter personal.

\footnotetext{
${ }^{1}$ En Madrid se ha desarrollado una situación extraordinaria en relación con la reivindicación de espacios socioculturales autogestionados que se están consolidando desde el año 2008.
} 
Sin embargo, según Boyer la clave de la memoria urbana colectiva está en los lugares de referencia, los espacios comunes que alcanzan un carácter simbólico para los ciudadanos y concluye que la memoria colectiva es la que une el espacio físico con las personas para transmitir valores y tradiciones.

Pero los lugares de referencia, al fin y al cabo, el espacio público de las ciudades, sufren una situación crítica. El lugar del soporte de la vida colectiva y cotidiana se comienza a ver, por parte de políticos e instituciones, como el espacio de la inversión para la elaboración de una imagen o una "marca" 2 .

El caso de la ciudad de Madrid a principios del siglo XXI ha sido paradigmático en este sentido, con la incorporación de "grandes proyectos" de altísimo coste que han situado la ciudad en el panorama arquitectónico y urbanístico internacional a costa de un enorme aumento de la deuda pública.

A esta situación se le añade la progresiva privatización del espacio público, asunto alarmante del que apenas se habla y que se traduce en la mercantilización de aceras, plazas e incluso de edificios públicos con fines comerciales. Sobre la privatización del espacio público dice Saskia Sassen,

(...) Todo ello pone de relieve la importancia de la verdadera creación del espacio público en un contexto en el que la privatización del espacio público y su utilización como arma se están radicalizando. He aquí un cambio de significados de la condición urbana (Sassen, 2012).

Esta nueva condición urbana produce consecuencias todavía más dramáticas a las que describe Christine Boyer en los años 90: observamos aceras sin bancos, mercadillos de venta de productos en plazas o construcciones efímeras publicitarias de cualquier producto comercial en nodos peatonales y de transporte público.

La realidad es que se pierde el uso cotidiano del espacio público en favor de un uso comercial que, además de negar su disfrute al vecino, destroza el paisaje urbano de la ciudad. Tal y como comenta Jordi Borja en su libro Ciudad y Ciudadanía:

La tentación de dejar el desarrollo urbano a la libre competencia y a los valores económicos inmediatos del mercado es muy peligroso, ya que el espacio público es especialmente rentable en términos sociales, culturales y civiles; pero

\footnotetext{
2 En los años 2000 se habla de Marca España, Marca Madrid, Marca Barcelona, etc.
} 
también lo es, a medio plazo, en términos políticos, la gobernabilidad, y económicos, generando atracción y creación de nuevas actividades.

El espacio público tiende fundamentalmente a la mezcla social, hace de su uso un derecho ciudadano de primer orden, así el espacio público debe garantizar en términos de igualdad la apropiación por parte de diferentes colectivos sociales y culturales, de género y edad (Borja, Muxí, 2003).

Esta rentabilidad a medio plazo provoca que las zonas históricas de las ciudades se vean afectadas además por el supuesto "turismo cultural" que, generalmente, produce una invasión de los centros históricos tanto de turistas como de recursos asociados al turismo. Este tipo de turismo no sólo perjudica a la imagen de la ciudad sino que además produce una importante alteración de la vida urbana de los ciudadanos residentes.

Es habitual desde finales del siglo XX medir en términos de impacto ambiental el turismo que afecta a los parajes naturales o a las costas de un país, pero es más difícil cuantificar hasta qué punto puede soportar un espacio urbano el turismo de masas. Quizá el umbral esté en el conflicto entre las dos formas principales de vivir la ciudad: como residente y como visitante. Cuando la ciudad no puede asumir los dos tipos de usuarios podemos hablar de una ciudad saturada.

Existe un turismo cultural que se refuerza con la transformación física de los centros urbanos para tematizarlos o caricaturizarlos con la inclusión de objetos típicos o la decoración zafia de su espacio público. En este sentido "la mercantilización urbana" se produce de una forma más amplia y dañina. Las "ciudades históricas producto" son cada vez más numerosas y el centro de la ciudad de Madrid se está transformando también en este sentido.

“La sociedad del espectáculo" (término acuñado por Guy Débord) parece estar consumiendo la memoria colectiva y las posibilidades del ciudadano de actuar como ese ciudadano activo (bios politikos) que ya reclamó Hannah Arendt a finales de los años 50. El hombre moderno, tal y como dice Débord, es demasiado espectador. La proliferación de "pseudoacontecimientos prefabricados" que denuncia se deriva de la falta de experiencia de sus propios acontecimientos. Ante la catastrófica situación y las perspectivas de no mejorar es necesario pensar en nuevas formas de relación con el espacio público para recuperar el vínculo como lugar de vida y experiencia comunitaria. 
Uno de los primeros pasos para estrechar el vínculo entre ciudad y ciudadanos debería ser el reconocimiento del interés de los ciudadanos en explorar su ciudad. Los ciudadanos deben valorar las posibilidades de sus ciudades a través del conocimiento sensible y de su experiencia subjetiva y personal.

Del mismo modo que el movimiento situacionista se adhería a los preceptos de denuncia que Guy Débord propone, también da un paso más allá en la propuesta de una aproximación espontánea hacia la ciudad a través de experiencias únicas y emotivas.

Los situacionistas proponen un modo de análisis relativo a través del concepto de "psicogeografía": un estudio subjetivo de la ciudad que se expresa a través de la elaboración de mapas de recorridos que proporcionan un vínculo más intenso del ciudadano con su ciudad. Esta aproximación se concreta en lo que llamarán "la deriva", término definido por Francesco Careri en su obra Walkscapes:

Después de la "visita" Dada, y de la "deambulación" surrealista, se acuña una palabra nueva: la dérive, una actividad lúdica colectiva que no sólo apunta hacia la definición de las inconscientes de la ciudad, sino que también se propone investigar, apoyándose en el concepto de psicogeografía, los efectos psíquicos que la ciudad produce en los individuos.

La deriva letrista desarrollaba la lectura subjetiva de la ciudad iniciada por los surrealistas, pero se proponía transformarla en un método objetivo de exploración de la ciudad: el espacio urbano era un terreno pasional objetivo, y no sólo subjetivo e inconsciente (Careri, 2002).

Los letristas van más allá de los surrealistas y aportan valor a "la experiencia urbana". Esta experiencia tiene suficiente potencia como para despertar los sentimientos dormidos del transeúnte urbano y puede ser algo tan sencillo como un atento paseo en la ciudad. Michel de Certeau habla de las míticas de lo que "hace andar":

$\mathrm{Al}$ vincular acciones y pasos, al relacionar sentidos y direcciones, estas palabras operan como un vaciamiento y un deterioro de su primera aplicación. Se convierten en espacios liberados, susceptibles de ser ocupados. Una rica indeterminación les permite, mediante un enrarecimiento semántico, la función de articular una segunda geografía, poética, sobre la geografía del sentido literal, prohibido o permitido. Insinúan otros viajes en el orden funcionalista e histórico de la circulación. (...) Lo que hace andar son las reliquias del sentido, y a veces sus desechos, los restos opuestos a las grandes ambiciones. Nadas o casi 
nadas simbolizan y orientan los pasos. Nombre que precisamente han dejado de ser "propios" (Certeau, 1980).

Lo lateral, lo escondido, los espacios olvidados son los lugares evocadores para los que realmente deciden explorar la ciudad.

Desde el movimiento letrista y situacionista, casi todas las aproximaciones a la práctica exploratoria de la ciudad han venido del mundo del arte. Urbanistas, sociólogos y geógrafos se acercan a la ciudad para diseccionar sus usos, su población, sus dinámicas o sus aspectos físicos o morfológicos. Sin embargo la experiencia inmediata, que busca la aparición de sentimientos en su diálogo individuo-ciudad propuesta por los letristas no tiene una continuidad en estas disciplinas. Es a través del arte y los artistas cuando "la experiencia urbana" tiene manifestación más clara.

La práctica deambulatoria consiste en una vivencia de la ciudad y su espacio público (a menudo camuflado, oculto, lateral) que se utiliza como marco exploratorio. Michel de Certeau llama a esta experiencia "geografía poética". La intensidad del hecho poético estrecha los vínculos entre los transeúntes y su espacio explorado. Esos lugares que, en palabras de Christine Boyer: “(...) deben aparecer ante nosotros como escenas trágicas, cómicas y satíricas de nuestro ordinario y extraordinario vivir cotidiano" (Boyer, 1996).

Ese vivir extraordinario podría ser proporcionado a partir de la experiencia de una ciudad en la que la búsqueda de "lo bello" fuera un objetivo. Como recuerda Baudelaire, la experiencia de lo bello puede ser una experiencia casi mística: "Stendhal, (...) se ha acercado a la verdad más que muchos otros, al decir que lo bello no es sino la promesa de la felicidad" (Baudelaire, 1863). Pero la búsqueda de la belleza y la necesidad de lo bello en la existencia humana es un tema que ha preocupado a un importante número de filósofos a lo largo de la historia reciente. La necesidad de considerar el arte de la era moderna y su origen a través de la filosofía resulta imperativa para justificar la idea de la importancia de la catarsis entre el arte y la ciudad a través del espacio público y sus habitantes.

En el contexto de comienzos del siglo XXI en el que se habla del fin del arte tradicional parece necesaria la refundación de unos principios nuevos que permitan evaluar el arte en sí mismo y su aportación a la sociedad actual. El arte expresado en cualquier formato pretende aportar una experiencia espiritual intensa, ya sea para proporcionar placer, sorpresa o rechazo. 
El arte no puede analizarse de forma separada del concepto de placer estético que, según J.M.Schaeffer, es la condición en la que una obra llena una función como objeto estético. Una experiencia estética es una experiencia de placer que no presupone el arte como cumplimiento de las funciones sociales, religiosas, políticas o existenciales. El placer estético se asemeja al placer físico en que se busca por sí mismo y reside en un estado de bienestar en el que nos tratamos de mantener el máximo tiempo posible. Esta idea tiene mucho en común con la definición de Stendhal que subraya Baudelaire asociando la idea de lo bello al concepto de felicidad.

Para Jean Marie Schaeffer conectar experiencia estética y el juicio de una representación no implica su intelectualización y la pérdida de las emociones. El placer dado en el juicio estético es una emoción, la obra de arte nos cautiva, nos fascina o nos interesa.

La sabiduría que encuentra sus fuentes en el arte no es diferente de aquella a la que llegamos a través de otras rutas cognitivas, en materia de experiencia diaria, filosófica o sabiduría científica: por eso es importante para nosotros y puede enriquecer nuestras vidas. El hecho del arte como necesario para compensar una sociedad exageradamente científica y consumista es una afirmación apoyada por los pensadores clásicos pero también por una sociedad contemporánea que valora las obras artísticas en términos económicos.

Según Schaeffer, Schopenhauer, Nietzsche o Heidegger se oponen al discurso científico con cierta nostalgia de lo que llaman "vida auténtica". De entre sus discursos podríamos concluir que el arte es necesario como contrapeso de una polémica interpretación de la vida común, asociada a prácticas rutinarias y funcionales. El arte como parte intrínseca de la vida puede tener su expresión más participativa o reivindicativa en el lugar que es común para todos: el espacio público. El diálogo entre este arte necesario y la ciudad y sus ciudadanos puede transformar la imagen de la ciudad y la memoria que tenemos de ella.

El arte como elemento distinto, como un intruso en la ciudad ayuda a enriquecer la "experiencia urbana". Si además se trata de un arte efímero en el que unas piezas se sustituyen por otras y algunas sirven de reclamo para llamar a otras nuevas, la experiencia todavía es más completa. La iconografía urbana alejada de los códigos publicitarios es un elemento que agradece el 
ciudadano incauto con ganas de explorar y encontrar espacios urbanos con entidad y carácter.

Tal y como reflexiona Saskia Sassen sobre lo que ella llama Urbanismo de código abierto:

(...) Mi preocupación aquí no son los espacios monumentalizados ni los ya construidos que, más que públicos, se definen como de acceso público. La creación de espacio público plantea cuestiones sobre la condición urbana actual en direcciones que los vastos espacios de la corona y del Estado o los sobredimensionados espacios de acceso público desconocen.

La labor de captar esta sutil cualidad que las ciudades generan y ponen de manifiesto y la tarea de crear espacio público en esta zona intermedia no son fáciles de llevar a cabo. La lógica de la funcionalidad no lo hará. No puedo evitar pensar que la creación del arte forma parte de la respuesta, sean fugaces representaciones e instalaciones públicas u otro tipo de escultura pública más duraderos, sea el arte específico del lugar/basado en la comunidad o esculturas nómadas que circulan por diversas localidades. Además, las nuevas tecnologías de redes plantean abiertamente la cuestión de crear en espacios pequeños y mediante las costumbres de la gente (...) (Sassen, 2012).

La respuesta del arte ante los problemas urbanos ya se está llevando a cabo, y de forma más potente, desde el arte efímero. En la voluntad de muchos artistas urbanos está el embellecer la ciudad, poner en valor espacios degradados o lugares olvidados. La permanencia de sus obras depende de numerosos factores pero existen numerosos ejemplos en los que se observa que cuanto más bella es la intervención artística, más posibilidades existen para que la intervención, concebida como efímera, se convierta en permanente ${ }^{3}$. La propuesta artística, sin embargo, no tiene fines solamente estéticos. Ciertas acciones creativas procuran ser herramientas para la recuperación de la memoria colectiva. El arte es necesario también para llenar ciertos vacíos identitarios tan frecuentes en los contextos urbanos postmodernos.

Existen experiencias muy interesantes de artistas que trabajan en lugares cuyos habitantes han perdido referencias o incluso partes de su memoria de-

\footnotetext{
${ }^{3}$ La artista madrileña Nuria Mora cuenta como intervenciones suyas en zócalos de edificios que han sobrevivido a obras de rehabilitación de fachadas donde los vecinos han decidido mantenerlas. La armonía de sus creaciones y la búsqueda de lo bello que le sirve de guía, como ella misma confiesa, son el secreto de su aceptación.
} 
bido a violentos cambios políticos o a desastres acontecidos durante revoluciones o guerras. El libro The (un) common place editado por Bartolomeo Pietromarchi compila un número importantes de trabajos artísticos en los que la memoria colectiva aparece como un tema recurrente.

La ciudad se ha convertido en una metáfora privilegiada de la experiencia en el mundo moderno. Con sus características originales, su mezcla de historias y culturas y su acogida de las tendencias globales que se ponen en cuestión de inmediato a través de sus singularidades locales, la ciudad se ofrece como un lugar real e imaginario, un mapa de fácil lectura. (...) la experiencia del espacio público no se desarrolla solamente en un nivel sincrónico sino también en un nivel diacrónico a través de memorias que se estratifican sobre los lugares y contribuyen a la construcción de la identidad específica del propio lugar, su Genius Loci, en el sentido de una elaboración cultural que es íntima y colectiva a la vez (Pietromarchi, 2005).

La ciudad, ciertamente, está configurada a través de una multiciplicidad de tiempos y, por tanto, la experiencia urbana es un hecho acumulativo. Por ese motivo la destrucción física de sectores de ciudad no sólo supone la pérdida de la edificación y su espacio físico, también produce pérdidas de la memoria y experiencias de muchos ciudadanos. La memoria colectiva y la pérdida o recuperación de la identidad es el leitmotif de un importante número de artistas de comienzos del siglo XXI con interés en lo urbano. El arte como complemento espiritual se justifica desde la búsqueda natural del ser humano de experiencias estéticas y su necesidad de recuperar y construir proyectos y espacios comunes.

Las expresiones artísticas en la ciudad actualmente exploran la potencialidad de la experiencia de la vida urbana con la incorporación de composiciones estéticas y la creación de nuevos lugares de encuentro, intercambio y comunicación que contribuyan a la generación o consolidación de proyectos comunitarios. La preocupación del arte sobre la memoria colectiva y la experiencia urbana es una realidad. La pregunta de si el arte puede contribuir a establecer un vínculo mayor entre el ciudadano y su ciudad a través de una vivencia urbana más intensa es uno de los asuntos que se pueden analizar a través de las expresiones de arte en la ciudad en la actualidad.

En la primera década del siglo XXI, el arte efímero urbano trasciende a los propios artistas y empieza a tenerse en cuenta como herramienta de expresión 
y visibilización con fines muy diversos. Las manifestaciones alrededor del arte efímero son dispares en función de su gestión, su origen y sus objetivos.

La potencia de la expresión del arte en la ciudad, espontáneo o gestionado de forma institucional comienza a ser patente a través de proyectos individuales y colectivos que corroboran que la necesidad de arte en la sociedad existe hoy en día del mismo modo que surgió desde los primeros procesos de fundación de las ciudades.

La transformación del espacio urbano a través del arte produce un paisaje urbano diferente. Ese paisaje urbano ha evolucionado a causa de la transformación del espacio público y de su adaptación a nuevos usos desde el origen de las ciudades hasta la ciudad del siglo XXI. Del mismo modo que el uso del espacio público ha evolucionado, también se han desarrollado las distintas formas de expresión del arte público. En la primera década del siglo XXI el arte en la ciudad ha adquirido mayor potencia como elemento efímero y cambiante. El arte efímero como expresión de la sociedad contemporánea se ha visto reflejado en numerosos ejemplos de arte público temporal y de arte urbano.

El interés que despiertan tanto las obras como los autores se debe, por un lado, a su valor estético pero también a su situación en lugares que son accesibles para todos los ciudadanos dentro de las ciudades. Desde el comienzo del siglo XXI el arte público temporal y el arte urbano han desencadenado procesos autónomos que comienzan a afectar al desarrollo de ciertas transformaciones urbanas con consecuencias a nivel local o global.

Hoy en día toda gran exposición de arte a nivel internacional tiene su repercusión en su ciudad de acogida a través de intervenciones efímeras en sus calles y plazas. El arte público temporal se utiliza como reclamo para señalar que algo extraordinario está sucediendo y la ciudad se invade con un lenguaje nuevo y atractivo. Algunas localidades viven su momento de gloria alrededor de estos eventos, que se repiten con una periodicidad rigurosa recibiendo a una multitud de peregrinos culturales (la Bienal de Venecia, la Trienal de Milan, Art Basel o la Bienal de Sao Paolo). Otra expresión de arte efímero se manifiesta desde la idea de "celebración de la ciudad" que se comienza a aprovechar por instituciones de numerosas localidades (La Nuite Blanche). Paralelamente aparece un tipo de festival más alternativo como son los festivales de arte urbano, con periodicidad anual o bianual (Bien Urbain, ASALTO, Weynwood Festival 
de arte Urbano de Barcelona, Urban Art Festival de Roma (Out Door), el Festival de Arte Urbano de México (allcitycanvas) o el Festival de Arte Urbano de Puerto Rico (losmuroshablan)

Con otros objetivos, el arte efímero se utiliza como instrumento de reflexión sobre situaciones urbanas. A través de la investigación artística se analizan circunstancias ciudadanas considerando aspectos sociales, económicos y físicos para fomentar la participación ciudadana (Idensitat o Madrid Abierto). Aparecen colectivos artísticos que se plantean el objetivo de crear vínculos y consolidar un sentimiento de identidad a través de la participación ciudadana con prácticas artísticas, generando proyectos comunitarios que se visibilizan en barrios populares (grupo Delborde en Argentina, Daniel Lima con proyectos como frente 3 de febreiro en Brasil, el grupo Nortec en México o el trabajo de Mónica Nádor). Estos movimientos artísticos, que parten de iniciativas espontáneas, provocan el interés de organismos público que plantean experiencias muy ambiciosas como los recientes de Planes de Mejora de Paisaje Urbano de gestión institucional.

El rico panorama del arte en la ciudad a principios del siglo XXI corrobora la necesidad de arte en una sociedad que ha sucumbido a la mercantilización de sus ciudades. Aunque el arte puede ser tratado como una mercancía más también es una herramienta de transformación urbana que puede contribuir a generar una ciudad más humana.

La necesidad de arte es también la necesidad de lo bello, esa búsqueda de la belleza es intrínseca al ser humano ya que, como dijo Stendhal, "lo bello no es sino la promesa de la felicidad" (Baudelaire, 1863).

\section{Bibliografía}

ARENDT, Hannah (2005): La condición humana. Paidós Ibérica.

BAUDELAIRE, Charles (2000): El pintor de la vida moderna. Colegio Oficial de Aparejadores y Arquitectos Técnicos. Murcia.

BENJAMIN, Walter (1999): Sobre algunos temas en Baudelaire. Leviatán. Madrid. BORJA, J. - MUXI, Z. (2003) El espacio público. Ciudad y ciudadanía. Electa. Barcelona.

BOYER, M. Christine (1996): The City of Collective Memory, its Historical Imagery an Architectural Entretainments. The MIT Press. Cambridge. 
CARERI, Francesco (2002): Walkscapes. Gustavo Gili. Barcelona.

De Certeau, Michel (2000): La invención de lo cotidiano. Artes de hacer. Cultura libre. México D.F.

DÉBORD, Guy (2009): La sociedad del espectáculo. S.L.U DOBLE J. Madrid.

JACOBS, Jane (2011): Muerte y vida de las grandes ciudades. Capitán Swing. Madrid.

NAdOR, M. (2012): Mónica Nador. Pinacoteca do Estado: Luciana Brito Galería.

PIETROMARCHI, Bartolomeo (2005): The (un)common place. Art, public space and urban aesthetics in Europe. Actar. Barcelona.

SADLER, Simon (1998): The situacionist city. MIT Press. Cambridge.

SASSEN, Saskia (2012): "Realizar intervenciones públicas en las grandes ciudades de hoy". En DíEZ, Jorge, Madrid Abierto 2009-2012. Asociación Cultural Madrid Abierto. Madrid.

SCHAEFFER, Jean Marie (2000): Art in the Modern Age. Princeton University Press. Princeton.

\section{Páginas web}

http://allcitycanvas.com/ Accedido Noviembre 2014.

http://bien-urbain.fr/fr/. Festival de arte urbano anual en la ciudad de Besançon. Accedido Septiembre 2014.

http://grupodelborde.blogspot.com.es/2007/04/da-7-casa-nmada.html. Accedido Septiembre 2014.

http://losmuroshablan.wordpress.com/ Accedido Noviembre 2014.

http://www.biennialfoundation.org/biennials/sao-paolo-biennialv/ Accedido Agosto 2014

http://www.festivalasalto.com/ Accedido Noviembre 2014.

http://www.finerats.com/2014/01/us-festival-dart-i-espai-de-barcelona/ Accedido Noviembre 2014.

http://www.idensitat.net/es/ "Idensitat es un proyecto de arte que investiga sobre las maneras de incidir en el ámbito del espacio público a través de propuestas creativas en relación con el lugar y el territorio desde una dimensión física y social.", Accedido en Septiembre 2014.

http://www.labiennale.org/it/arte/index.html Accedido Agosto 2014

http://www.triennale.it/it/ Accedido Agosto 2014

http://wynwoodmiami.com/home.php. Festival de arte urbano en la ciudad de Miami

Accedido Agosto 2014.

https://www.artbasel.com/ Accedido Agosto 2014 